\title{
Relationship between bile acid malabsorption and pancreatic insufficiency in cystic fibrosis
}

\author{
A. M. WEBER ${ }^{1}$, C. C. ROY, L. CHARTRAND, G. LEPAGE, O. L. DUFOUR, \\ C. L. MORIN, AND R. LASALLE
}

From the Department of Pediatrics, Hôpital Sainte-Justine, and the University of Montreal, Montreal, Canada

SUMMARY Bile acid loss $\left(\mathrm{mg} / \mathrm{m}^{2} 24 \mathrm{~h}\right)$ in the stools of 43 cystic fibrosis (CF) children with pancreatic insufficiency was $751 \cdot 1 \pm 48 \cdot 3$, while that of six without clinical evidence of pancreatic disease $(133 \cdot 4$ $\pm 15 \cdot 9)$ did not differ from values in 25 controls $(109 \cdot 8 \pm 9 \cdot 8)$. There was a good correlation between the degree of bile acid (BA) and fat sequestration. Concomitant changes in bile acid and fat loss were observed in one group of six patients studied on and off pancreatic enzymes as well as in a second group of seven children treated with pancreatic supplements and maintained on a normal diet followed by a low fat diet supplemented with medium chain triglycerides. Administration of NA bicarbonate led to a significant decrease in fat loss $(15.8 \pm 2.7 \rightarrow 10.3 \pm 1.9)$ without any simultaneous change in bile acid excretion $(533 \cdot 1 \pm 58 \cdot 3 \rightarrow 500 \cdot 4 \pm 58 \cdot 6)$. Qualitative bile acid patterns in controls, in infants after an ileal resection, and in patients with CF or with coeliac disease showed that the percentage of primary BA followed closely the total amount excreted except in situations where antibiotics were administered. The exact mechanism for the increased loss of BA in CF is unknown. It is found in all age groups and is related to the presence and degree of pancreatic insufficiency. The possibility that unhydrolysed triglycerides may interfere with the intestinal absorption of bile acid needs further confirmation.

In a previous paper (Weber et al., 1973), we found that bile acid (BA) loss in the stools of 24 untreated children with pancreatic insufficiency secondary to cystic fibrosis (CF) was markedly increased. Mean values in the $C F$ population were more than seven times those in a control group, and comparable with the excretion in infants after an ileal resection. Kinetic studies have since confirmed that BA turnover in CF is accelerated and compatible with an interruption of the enterohepatic circulation (Watkins et al., 1974; Weber et al., 1975).

The clinical implications of our findings and their possible impact on management have prompted the present study designed to characterize further the BA malabsorption, and to explore its pathogenesis. The data reported concern a larger number of patients with a wider age range, $\mathrm{CF}$ children without pancreatic insufficiency, and the response of a small group to various dietary and therapeutic interventions.

${ }^{1}$ Address for reprint requests: Dr. Andrée Weber, Hôpital SainteJustine, 3175 St. Catherine Road, Montreal H3T, 1C5, Que. Canada.

Received for publication 30 January 1976

\section{Methods}

PATIENTS

The 74 patients included in this study were divided into 25 normal controls and 49 untreated CF children. Among them, 18 controls and $24 \mathrm{CF}$ patients have already been reported on (Weber et al., 1973). Six of the 25 CF children, who were more recently investigated, had no clinical evidence of exocrine pancreatic insufficiency and none had steatorrhea. Twelve of these 25 patients were studied during the course of an initial diagnostic workup. Most of the others were evaluated on an outpatient basis, including the seven new controls who were volunteers without any clinical evidence of pancreatic, hepatic, or gastrointestinal disease.

CF patients with evidence of liver involvement were not included in the study. The $49 \mathrm{CF}$ children had normal liver function tests including a BSP excretion. The oldest patient (20 years) presented with cholelithiasis.

A small group of CF children with pancreatic insufficiency were studied under the following dietary and therapeutic regimens: (1) six patients during 
pancreatic enzyme therapy and seven days after discontinuation; (2) seven patients at three different intervals on a fixed amount of enzymes (Table 1). The number of capsules was calculated on the basis of one per gram of stool fat above the value of $4 \mathrm{~g}$ per $24 \mathrm{~h}$. The study was repeated after 10 days of added sodium bicarbonate $\left(15 \mathrm{~g} / 1 \cdot 73 \mathrm{~m}^{2} .24 \mathrm{~h}\right)$. The tablets $(600 \mathrm{mg})$ were administered with the pancreatic enzyme supplements before each of the three main meals. The last study followed a 10 day period during which the children were maintained on a low fat diet $(7 \%$ of caloric intake) supplemented with medium chain triglycerides $(28 \%$ of caloric intake) supplied as oil (MCT oil, Mead Johnson Canada Ltd.).

The investigation consisted of a three day stool collection. Faeces were collected between markers, as previously described (Weber et al., 1973). The older patients used plastic bags designed to fit camping commodes. Each stool specimen was immediately frozen. Unless otherwise specified, antibiotics were discontinued for at least three weeks before the investigation. For home collections, parents were met in groups or individually. The aim of the project and the method of collection were explained. Phone contacts were then established during the period of investigation in order to avoid any error in the procedure.

Stool fat $(\mathrm{g} / 24 \mathrm{~h})$ was determined following the method of Van de Kamer $e$ t al. (1949). Chymotrypsin (Bonin et al., 1973) was measured in faeces of CF patients including all those without steatorrhea. The enzymatic method served for the determination of faecal BA expressed as $\mathrm{mg}$ per $\mathrm{m}^{2} 24 \mathrm{~h}$ (Weber et al., 1972). With the use of a gas liquid chromatograph (Weber et al., 1973), qualitative BA patterns were obtained in a certain number of children with $\mathrm{CF}$ and compared with those found in four infants after an ileal resection, in four untreated cases of coeliac disease, and in eight controls.

\section{Results}

The average age of the 43 untreated CF children

\begin{tabular}{llll}
\hline $\begin{array}{l}\text { Periods } \\
\text { of study }\end{array}$ & $\begin{array}{c}\text { Enzyme } \\
\text { therapy }\end{array}$ & $\begin{array}{l}\text { Sodium } \\
\text { bicarbonate }\end{array}$ & Diet \\
\hline I & + & - & Normal \\
II & + & + & Normal \\
III & + & - & Low fat and \\
& & & MCT
\end{tabular}

Table 1 Dietary and therapeutic regimens followed by seven $C F$ patients

*Cotazym Organon Inc., Toronto Canada.

The study was completed for each child over a period of one to six months. with steatorrhea was 3.9 years (range $2 / 12$ to 20 years), while that of the 25 controls was $5 \cdot 2$ years (range 4/12 to 15 years). Figure 1 shows that there was no correlation between the age and the degree of bile acid loss. In the older group of eight CF children (mean age 12.1 years) the magnitude $(\overline{\mathrm{X}} \pm \mathrm{SEM})$ of bile acid sequestration (610.3 \pm 98.2) was comparable with that $(787.3 \pm 53.5)$ of the $35 \mathrm{CF}$ patients younger than 6 years (mean 1.8 years). Similarly, the bile acid excretion in the controls varied little $(111 \pm 11$ versus $106 \cdot 7 \pm 22 \cdot 3)$ between the younger group (mean 2.4 years) of 18 and the older group (mean 11.8 years) of seven children.

In 43 untreated $\mathrm{CF}$ children with pancreatic insufficiency (Fig. 2) faecal fat (17.0 \pm 1.6$)$ and BA $(751 \cdot 1 \pm 48 \cdot 3)$ were significantly $(P<0.001)$ different from the values of $1 \cdot 4 \pm 0 \cdot 2$, and $109 \cdot 8 \pm 9 \cdot 8$, found in the 25 controls. However, the six CF patients without steatorrhea $(2 \cdot 5 \pm 0.2)$ excreted amounts of BA $(133.4 \pm 15.9)$ which compared with the values of normal controls. The scatter diagram achieved by plotting faecal BA (mg/24 h) as a dependent variable versus fat $(\mathrm{g} / 24 \mathrm{~h})$ as an independent variable, showed a linear relationship. The goodness of fit of these data to the regression line $(P<0.01)$ and the correlation coefficient $(r 0.66)(\mathrm{P}<0.001)$ were both significant.

Figure 3 shows the influence of enzyme therapy individually for each of the patients. A significant increase $(\mathrm{P}<0.001)$ in faecal BA $(543.5 \pm 72.1$ to $999.9 \pm 202)$ and fat $(8.9 \pm 1 \cdot 1$ to $20.5 \pm 3.8)$ was found when enzymes were withheld. After introducing a calculated amount of enzymes in seven patients (Fig. 4), the addition of sodium bicarbonate induced a further significant $(P<0.01)$ drop in steatorrhea $(15.8 \pm 2 \cdot 7$ to $10 \cdot 3 \pm 1.9)$ without any simultaneous change in BA excretion (533.1 \pm 58.3 to $500 \cdot 4 \pm 58 \cdot 6$ ).

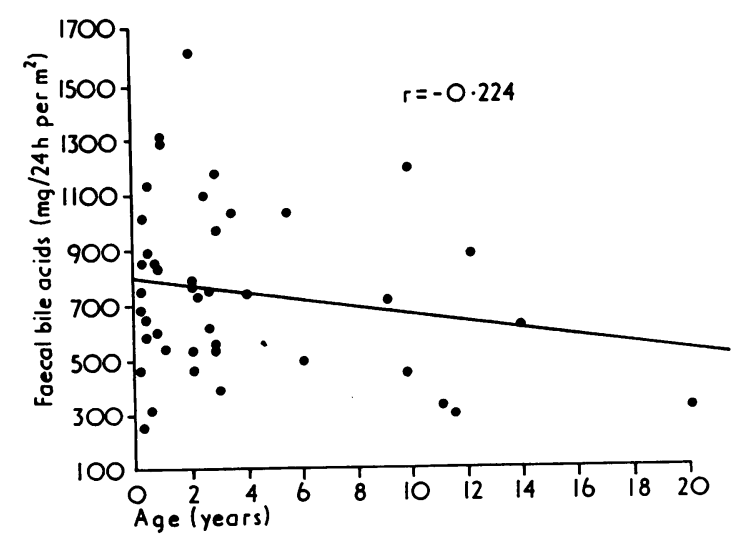

Fig. 1 Correlation between age and faecal $B A$ excretion in 43 untreated CF children. 


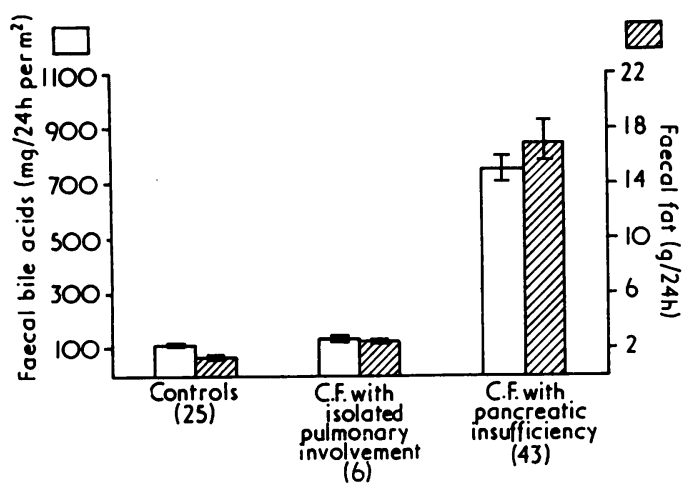

Fig. 2 Faecal fat and BA excretion in controls and in $C F$ children with and without pancreatic insufficiency.

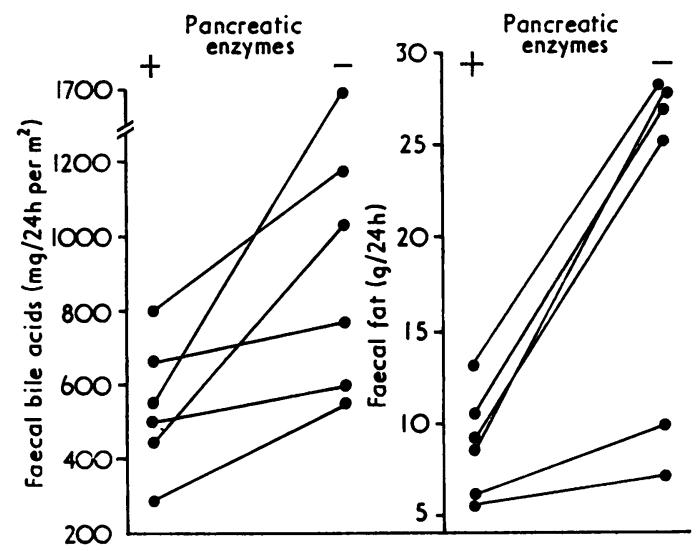

Fig. 3 Variation of faecal fat and BA excretion induced by pancreatic enzyme therapy in six CF children.

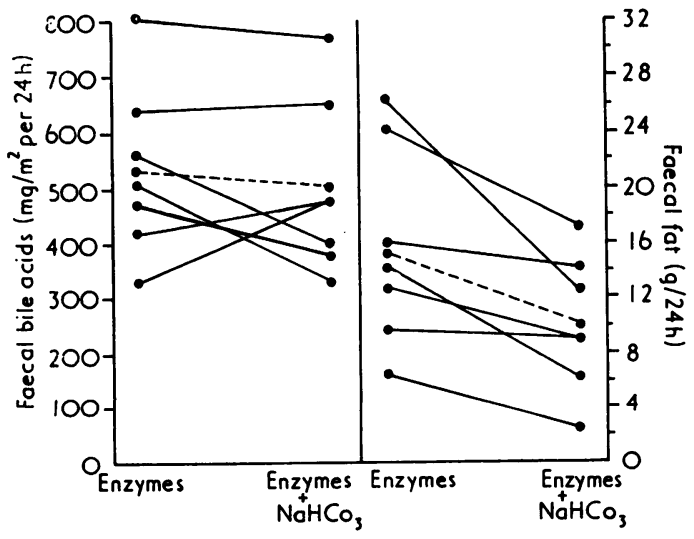

Fig. 4 Effect of sodium bicarbonate on faecal fat and $B A$ excretion in seven $C F$ children treated with pancreatic enzymes.
The administration of a low fat diet rich in MCT brought about a concomitant decrease in fat $(15.8 \pm$ $2 \cdot 7$ to $11.0 \pm 2.0)$ and BA $(533.1 \pm 58.3$ to $457.7 \pm$ $77 \cdot 8$ ). In both cases, statistical significance was not achieved because, as seen in Fig. 5, two patients (R.B. and C.R.) unexpectedly lost more fat and BA under the regimen. Patient R.D. was the only one in whom the relationship between these parameters did not hold.

Qualitative BA patterns are compared in Table 2. The percentage of primary BA was increased in untreated CF patients $(58.5 \pm 4.0)$ and was similar to the value found in patients who underwent an ileal resection $(60 \cdot 2 \pm 4 \cdot 1)$. Antibiotic therapy in CF was followed by a further increase (72.7). Enzyme therapy induced a decrease in the percentage of primary BA $(43.8 \pm 6 \cdot 0)$ but it was still much higher than in controls $(13 \cdot 5 \pm 3 \cdot 2)$. Adding sodium bicarbonate $(31.0 \pm 6.1)$ or MCT $(31.6 \pm 4.5)$ to the enzyme supplements resulted in a small additional variation. CF patients without pancreatic insufficiency excreted $27 \% \pm 5.9$ of primary BA, a value still different from those of controls and coeliac children (12.4 $\pm 3 \cdot 6)$.

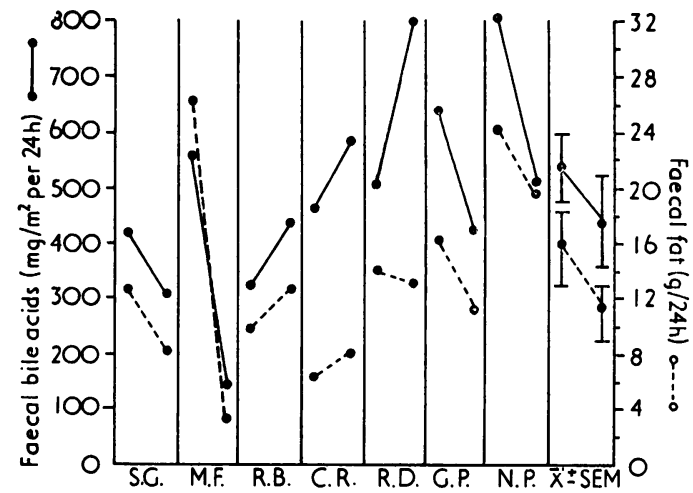

Fig. 5 Influence of a low fat diet supplemented with $M C T$ on the faecal fat and BA excretion of seven $C F$ children treated with pancreatic enzymes.

\section{Discussion}

The present findings confirm our previous observations that faecal BA excretion is increased in CF. The values obtained in this study from a greater number of older patients without liver disease show that this BA loss is persistent. In contrast, CF children without steatorrhea excrete normal amounts. BA malabsorption in CF is therefore unlikely to be associated with an intestinal defect specific for the mutant gene 


\begin{tabular}{lllll}
\hline Disease & Patients (no.) & Conditions & $\begin{array}{l}\text { Faecal BA excretion } \\
\left(\mathrm{mg} / \mathrm{m}^{2} / 24 \mathrm{~h}\right)\end{array}$ & Primary BA* in faeces (\%) \\
\hline Cystic fibrosis & 6 & No therapy & $999 \cdot 9 \pm 202$ & $58 \cdot 5 \pm 4 \cdot 0$ \\
& 6 & Enzymes & $543 \cdot 5 \pm 72 \cdot 1$ & $30 \cdot 6 \pm 5 \cdot 3$ \\
& 7 & Enzymes (C) $\dagger$ & $533 \cdot 1 \pm 58 \cdot 3$ & $43 \cdot 8 \pm 6 \cdot 0$ \\
& 7 & (C) and Na bicarbonate & $500 \cdot 4 \pm 58 \cdot 6$ & $31 \cdot 0 \pm 6 \cdot 1$ \\
& 7 & (C) and MCT diet & $457 \cdot 7 \pm 77 \cdot 8$ & $31 \cdot 6 \pm 4 \cdot 5$ \\
Ileal resection & 2 & Antibiotics & $707 \cdot 5$ & $72 \cdot 7$ \\
Coelic disease & 6 & No steatorrhea & $133 \cdot 4 \pm 15 \cdot 9$ & $27 \cdot 0 \pm 5 \cdot 9$ \\
Controls & 4 & No therapy & $1250 \cdot 3 \pm 155 \cdot 1$ & $60 \cdot 2 \pm 4 \cdot 1$ \\
& 8 & No therapy & $102 \cdot 4 \pm 45 \cdot 8$ & $11 \cdot 8 \pm 4 \cdot 6$ \\
\hline
\end{tabular}

Table 2 Comparison between quantitative and qualititative faecal BA patterns

${ }^{*}$ Cholic + chenodeoxycholic acids $(\mathrm{mg}) \times 100 /$ total BA.

$\dagger(C)$ : calculated amount of pancreatic enzyme supplements.

and occurs only in the presence of pancreatic insufficiency.

The extent of the BA loss varies with the degree of pancreatic insufficiency as shown by its correlation with faecal fat. The concomitant improvement of BA and fat malabsorption documented in patients receiving pancreatic enzymes also supports this relationship and suggests that unhydrolysed triglycerides might interfere with $\mathrm{BA}$ absorption. The fact that steatorrhea of coeliac disease is not associated with BA malabsorption also favours this hypothesis (Weber et al., 1973).

Medium chain triglycerides (MCT) have been used by a number of workers in CF and were reported to be beneficial in decreasing fat losses (Kuo and Huang, 1965; Gracey et al., 1970). Although two of our patients showed increased steatorrhea when MCT were fed, it is worth noting that, in all but one child, the concomitant variation in BA and fat excretion was still present. One could question whether the long chain triglycerides (LCT) or the MCT in the diet was responsible for the variation in BA excretion. A direct influence of MCT on BA absorption has been suggested (Holt, 1971). However, in the Rhesus monkey, a fat-free diet was shown to be more effective in decreasing BA excretion than a MCT diet. A diet rich in LCT increased BA losses (Redinger et al., 1973) and it has been recently reported that long chain fatty acids inhibit BA absorption (Ammon and Phillips, 1974). It therefore appears that the presence or absence of LCT in the diet seems to be an important factor influencing BA metabolism.

The beneficial influence of sodium bicarbonate supplements on fat absorption has been previously reported in exocrine pancreatic insufficiency. (Haro and Faloon, 1964; Kattwinkel et al., 1972). Possible reasons are: (1) prevention of pancreatic enzyme supplements from gastric inactivation (Heizer $e t$ al., 1965); (2) increase in duodenal pH content (Veeger et al., 1962) allowing-optimal activity of pancreatic lipase (Go et al., 1970) - and/or facilitated incorporation of fatty acids into micelles (Borgström, 1967) - and/or enhanced fatty acids absorption at the mucosal level (Annegers, 1953). In this study, the drop in fat excretion was not followed by a concomitant decrease in BA when sodium bicarbonate was introduced. If unhydrolysed fat is the factor to be incriminated in the BA malabsorption found in $\mathrm{CF}$, we would suspect that the major effect of bicarbonate therapy is an increased micellar incorporation of lipolytic products leading to a better absorption. The influence of duodenal $\mathrm{pH}$ on fat hydrolysis and solubilization in CF has been reported in preliminary form (Ricour and Rey, 1972). In view of our results with bicarbonate, further investigation appears warranted.

Qualitative BA patterns were examined in the hope of establishing a relationship with the total amount excreted. Our data found in patients after ileal resection are in agreement with previous reports (Mallory et al., 1973; Hofman and Poley, 1972) showing an increased percentage of primary BA. Similar patterns in CF further confirm that, whenever excessive losses are found, a higher percentage of primary BA is excreted. A likely explanation is that high concentrations of bile acids in the lower intestinal tract lead to a reduction in the ability of the microflora to transform BA into its usual metabolites (Floch et al., 1971) since both in vitro (Eastwood et al., 1972) and in vivo (Fiasse et al., 1974) studies have shown that BA inhibit the growth of intestinal bacteria, particularly anaerobes.

The possibility that the absence of conversion of primary BA into secondary forms could account for their malabsorption can be dismissed. A decreased excretion of BA is found in germ-free animals where no secondary BA are present. Furthermore, in the two patients whose flora were modified by antibiotics, the percentage of primary BA was high and yet their total excretion was in the range of other $\mathrm{CF}$ 
children. The preponderance of primary BA in CF is probably not totally explained by the large quantitative loss. The data in children without fat malabsorption suggest that the repeated use of antibiotics in CF with attendant changes in the intestinal microflora could lead to an increased percentage of primary BA despite normal total amounts in the stools.

This investigation would not have been possible without the concern and excellent collaboration of the CF patients and their parents. We wish to acknowledge the financial support of the Medical Research Council of Canada, the Canadian Cystic Fibrosis and the Justine Lacoste-Beaubien Foundations. Dr. A. Weber was supported by a Research fellowship from Le Conseil de la Recherche Médicale du Québec.

\section{References}

Ammon, H. V., and Phillips, S. F. (1974). Inhibition of ileal water absorption by intraluminal fatty acids. Journal of Clinical Investigation, 53, 205-210.

Annegers, J. H. (1954). Function of pancreatic juice and of bile in assimilation of dietary triglyceride. Archives of Internal Medicine, 93, 9-22.

Bonin, A., Roy, C. C., Lasalle, R., Weber, A., and Morin, C. L. (1973). Fecal chymotrypsin: a reliable index of exocrine pancreatic function in children. Journal of Pediatrics, 83, 594-600.

Borgström, B. (1967). Partition of lipids between emulsified oil and micellar phases of glyceride-bile salt dispersions. Journal of Lipid Research, 8, 598-608.

Eastwood, M. A., Findlay, J. M., MacRae, R., and Mitchell, W. D. (1972). Why are primary bile acids present in cholerhoeic diarrhoea ? Gut, 13, 845-846. (Abstract.)

Fiasse, R., Eyssen, H., Leonard, J. P., and Dive, C. (1974). Intestinal absorption and fecal bile acid analysis in Crohn's disease and after ileal resection. A study of 106 patients. III ${ }^{d}$ International Congress of Gastroenterology, Mexico City (Abstract).

Floch, M. H., Gershengoren, W., Elliott, S., and Spiro, H. M. (1971). Bile acid inhibition of the intestinal microfloraa function for simple bile acids ? Gastroenterology, 61, 228233.

Go, V. L. W., Poley, J. R., Hofmann, A. F., and Summerskill, W. H. J. (1970). Disturbances in fat digestion induced by acidic jejunal $\mathrm{pH}$ due to gastric hypersecretion in man. Gastroenterology, 58, 638-646.

Gracey, M., Burke, V., and Anderson, C. M. (1970). Medium chain triglycerides in paediatric practice. Archives of Disease in Childhood, 45, 445-452.
Haro, E. N., and Faloon, W. W. (1964). The effect of bicarbonate on pancreatic enzyme activity. Clinical Research, 12, 207.

Heizer, W. D., Cleaveland, C. R., and Iber, F. L. (1965). Gastric inactivation of pancreatic supplements. Bulletin of the Johns Hopkins Hospital, 116, 261-270.

Hofman, A. F., and Poley, J. R. (1972). Ratio of bile acid malabsorption in pathogenesis of diarrhea and steatorrhea in patients with ileal resection. Gastroenterology, 62, 918934.

Holt, P. R. (1971). Medium chain triglycerides. Disease-aMonth, June, p. 19.

Kattwinkel, J., Agus, S. G., Taussig, L. M., di Sant'Agnese, P. A., and Laster, L. (1972). The use of L-arginine and sodium bicarbonate in the treatment of malabsorption due to cystic fibrosis. Pediatrics, 50, 133-137.

Kuo, P. T., and Huang, N. N. (1965). The effect of medium chain triglyceride upon fat absorption and plasma lipid and depot fat of children with cystic fibrosis of the pancreas. Journal of Clinical Investigation, 44, 1924-1933.

Mallory, A., Kern, F., Smith, J., and Savage, D. (1973). Patterns of bile acids and microflora in the human small intestine. 1. Bile acids, Gastroenterology, 64, 26-33.

Redinger, R. N., Hermann, A. H., and Small, D. M. (1973). Primate biliary physiology, X: effects of diet and fasting on biliary lipid secretion and relative composition and bile salt metabolism in the rhesus monkey. Gastroenterology, 64, 610-621.

Ricour, C., and Rey. J. (1972). Étude de l'hydrolyse et de la solubilisation micellaire des graisses par perfusion intestinale chez l'enfant normal et au cours des déficits de sécrétion biliaire et lipasique. Archives Françaises de Pédiatrie, 29, 668-669. (Abstract).

Van de Kamer, J. H., Huinink, H. Ten Bokkel, Weyers, W. A. (1949). Rapid method for the determination of fat in feces. Journal of Biological Chemistry, 177, 347-355.

Veeger, W., Abels, J., Hellemans, N., and Nieweg, H. O. (1962). Effect of sodium bicarbonate and pancreatin on the absorption of vitamin $B_{12}$ and fat in pancreatic insufficiency. New England Journal of Medicine, 267, 1341-1344.

Watkins, J. B., Tercyak, A. M., Szczepanik, P., and Klein, P. D. (1974). Bile salt kinetics in cystic fibrosis. Gastroenterology, 67, 835. (Abstract).

Weber, A. M., Chartrand, L., Doyon, G., Gordon, S., and Roy, C. C. (1972). The quantitative determination of fecal bile acids in children by the enzymatic method. Clinica Chimica Acta, 39, 524-531.

Weber, A. M., Roy, C. C., Lepage, G., Chartrand, L., and Lasalle, R. (1975). Interruption of the enterohepatic circulation of bile acids in cystic fibrosis. Gastroenterology, 68, 1066. (Abstract).

Weber, A. M., Roy, C. C., Morin, C. L., and Lasalle, R. (1973). Malabsorption of bile acids in children with cystic fibrosis. New England Journal of Medicine, 289, 10011005. 